\title{
Comparisons between PW Doppler system and enhanced FM Doppler system
}

\author{
Wilhjelm, Jens E.; Pedersen, P. C.
}

Published in:

Proceedings of the IEEE Ultrasonics Symposium

Link to article, DOI:

10.1109/ULTSYM.1995.495850

Publication date:

1995

Document Version

Publisher's PDF, also known as Version of record

Link back to DTU Orbit

Citation (APA):

Wilhjelm, J. E., \& Pedersen, P. C. (1995). Comparisons between PW Doppler system and enhanced FM Doppler system. In Proceedings of the IEEE Ultrasonics Symposium (Vol. Volume 2, pp. 1549-1552). IEEE. https://doi.org/10.1109/ULTSYM.1995.495850

\section{General rights}

Copyright and moral rights for the publications made accessible in the public portal are retained by the authors and/or other copyright owners and it is a condition of accessing publications that users recognise and abide by the legal requirements associated with these rights.

- Users may download and print one copy of any publication from the public portal for the purpose of private study or research.

- You may not further distribute the material or use it for any profit-making activity or commercial gain

- You may freely distribute the URL identifying the publication in the public portal 


\title{
Comparisons Between PW Doppler System and Enhanced FM Doppler System
}

\author{
J. E. WILHJELM \& P. C. PEDERSEN $\ddagger$ \\ EI, Tech. Univ. of Denmark, Bldg. 349, DK-2800 Lyngby, Denmark. \\ ${ }^{\ddagger}$ Elec. and Comp. Eng. Dept., Worcester Polytechnic Institute, Worcester, MA 01609, USA.
}

\begin{abstract}
This paper presents a new implementation of an echo-ranging FM Doppler system with improved performance, relative to the $F M$ Doppler system reported previously. The use of long sweeps provides a significant reduction in peak to average power ratio compared to pulsed wave $(P W)$ emission. A $P W$ Doppler system exploits the direct relationship between arrival time of the received signal and range from the transducer. In the FM Doppler systems, a similar relationship exists in the spectral domain of the demodulated received signals, so that range is represented by frequency. Thus, a shift in location of moving scatterers between consecutive emis. sions corresponds to a frequency shift in the spectral signature. The improvement relative to the earlier version of the FM Doppler system is attained by utilizing cross-correlation of real spectra rather than of magnitude spectra for assessing flow velocity. This approach requires a priori knowledge of the envelope of the received sweep from a point scatterer.
\end{abstract}

\section{INTRODUCTION}

The non-invasive assessment of blood flow with PW Doppler ultrasound is today a standard technique in hospitals and clinics. However, to reduce the high peak power of the emitted PW signal, but at the same time maintain a wide bandwidth, Doppler systems emitting coherent, repetitive frequency modulated (FM) signals have been developed. ${ }^{[3.4]}$ This paper presents an improved version of the FM frequency shift measurement (FM-fsm) Doppler technique $e^{[5]}$ which in several respects is analogous to the $\mathrm{PW}$ time shift measurement, ${ }^{11}$ PW-tsm, technique. Simultaneous emission and reception is generally utilized in the FM Doppler system, thus requiring a dual transducer system.

\section{2 . EMITTED AND RECEIVED SIGNAL}

For a given measurement of a velocity profile, the entire emitted signal consists of $N$ sweep signals with a Gaussian envelope, denoted $n=[0 ; N-1]$. The time interval between consecutive sweeps is $T_{r}$. Each sweep signal has the form

$g_{t}(t)=\exp \left[-2\left(\frac{\alpha}{t_{m}}\right)^{2}\left(t-\frac{t_{m}}{2}\right)^{2}\right] \cos \left(2 \pi f_{1} t+\pi S_{0} t^{2}\right)$ over the interval $0<t<t_{m}$. The parameter $t_{m}$ is the length of the signal (typical values ranges from $50 \mu \mathrm{s}$ to $200 \mu \mathrm{s}$ ), $\alpha$ is a form factor for the Gaussian envelope (normally $\alpha$ $=3), f_{t}$ is the start frequency at $t=0$ and $S_{0}$ is the sweep rate in $\mathrm{Hz} / \mathrm{s}$. If the stop frequency at $t=t_{m}$ is called $f_{2}$, then $S_{0}=\left(f_{2}-f_{1}\right) / t_{m}$. In this paper, the emitting and receiving transducers are assumed ideal, except for a bandlimited frequency response that is modelled directly into (1) by the Gaussian envelope.

Assuming that the received sweep from a scatterer moving axially away from the transducer with speed $v$ and located at range $d^{(0)}$ at $t=0$ and $n=0$ can be written as an amplitude modulated, time delayed and Doppler compressed replica of the emitted signal, the $n$th received signal can be written as

$$
\begin{aligned}
g_{r}^{(n)}(t) & =r g_{t}\left(\beta\left(t-2 \frac{d^{(n)}}{c-v}\right)\right) \\
& \cong r \exp \left[-\alpha_{G}\left(t-t^{(n)}-\frac{t_{m}}{2}\right)^{2}\right] \\
& \times \cos \left(2 \pi f_{1}\left(t-t^{(n)}\right)+\pi S_{0}\left(t-t^{(n)}\right)^{2}\right)
\end{aligned}
$$

where $t^{(n)} \leq t \leq t^{(n)}+t_{m}$ and

$$
t^{(n)}=\frac{2 d^{(n)}}{c}=2 \frac{d^{(0)}+n \nu T_{r}}{c}
$$

In (2), $r$ is the scattering coefficient of the scatterer, $\beta=$ $(c-v) /(c+v)$ is the Doppler compression factor and $\alpha_{G}=$ $2\left(\alpha / t_{m}\right)^{2}$. As the scattering model used in this paper is assumed linear, the results for the multi-scatterer situation can be found by use of superposition.

\section{3 . FM- $f \boldsymbol{s} m$ SIGNAL PROCESSING}

The block diagram for FM- $f s m$ Doppler system is shown in Figure 1. The received signal in the FM- $f s m$ Doppler system is basically a linear sweep signal which must be demodulated with a reference sweep signal in quadrature in order to establish a unique range-frequency relationship analogous to the range-time relationship known from PW excitation. The result of the demodulation, after lowpass filtering (LPF) and Fourier transform, is called the $f s m$ spectrum ${ }^{[4]}$ and denoted $\tilde{G}_{a}^{(n)}(f)$. 


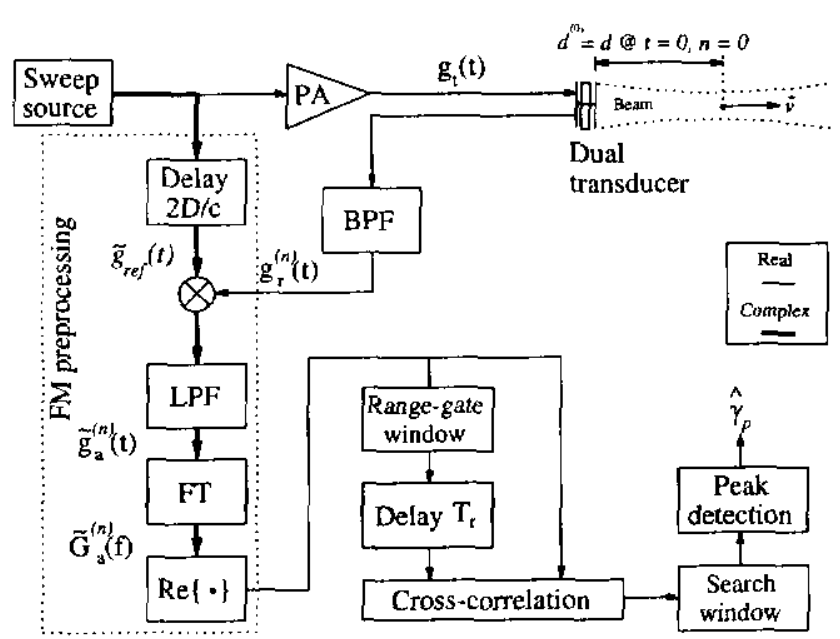

Figure 1 Block diagram of FM-fsm signal processing.

An example of the emitted sweep signal is given in Figure 2(a), while two consecutive received signals from one moving particle are presented in Figure 2(b-c). The constant amplitude reference sweep has the same start frequency and sweep rate as the emitted signal, but time delayed $t_{s}=2 D / c$ where $D$ is the measurement range.

For a single scatterer, the demodulated signal is a tone burst with Gaussian envelope and duration $t_{m}$. The mean frequency of this tone burst is proportional to the axial displacement of the particle from $D{ }^{[4]}$ Two consecutive demodulated signals are shown in Figure 2(d) and (e). Next, the demodulated signal is Fourier transformed into the frequency domain, with a temporal zero reference of
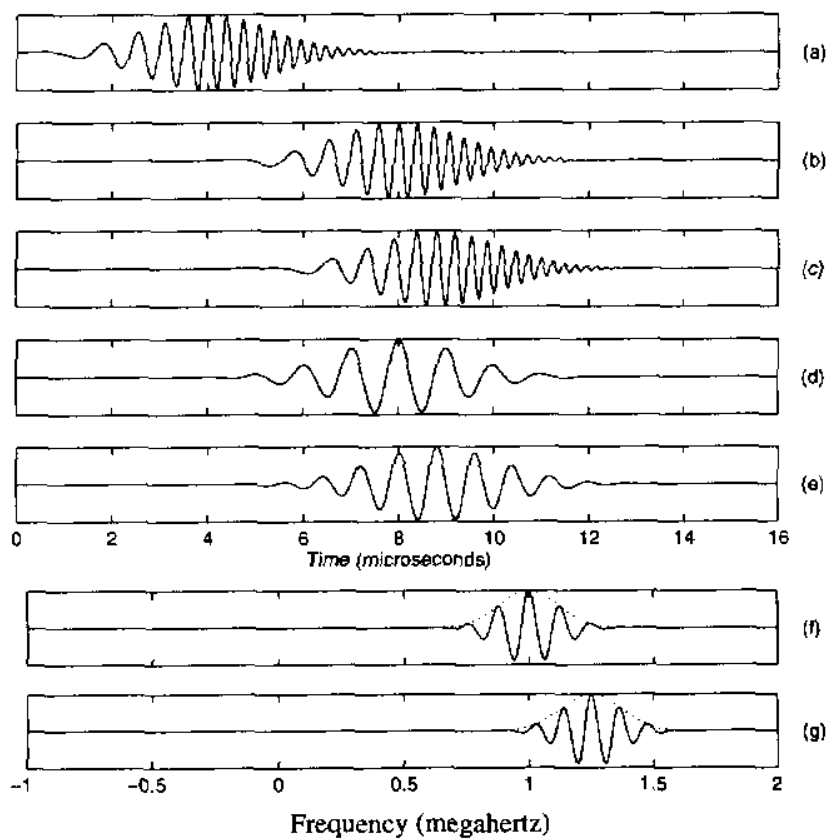

Figure 2 Signals and spectra of the FM- $f s m$ Doppler system. See text for details. In (f) and (g), (-) and ( (.).), corresponds to real part and magnitude, respectively. $t=t_{s}$. Figure 2(f) and (g) show the real and magnitude parts of two consecutive $f s m$ spectra. The $f s m^{[4]}$ spectrum is analogous to the received signal due to $\mathrm{PW}$ excitation and is processed in roughly the same fashion. As will be shown, consecutive fsm magnitude spectra are identical in shape, but shifted in frequency. In contrast, the phase function changes from one $f s m$ spectrum to the next, causing the real part of consecutive fsm spectra to differ both in frequency and in shape of the spectral waveform. The $f s m$ spectrum, called $\tilde{G}_{a}^{(n)}(f)$, can be shown to be ${ }^{\mid s]}$

$$
\begin{aligned}
& \tilde{G}_{a}^{(n)}(f)=r \sqrt{\frac{\pi}{\alpha_{G}}} \exp \left[-\frac{n^{2}}{\alpha_{G}}\left(f_{a}^{(n)}-f\right)^{2}\right] \\
& \times \exp \left[j 2 \pi\left(t_{d}^{(n)}+\frac{t_{m}}{2}\right)\left(f_{a}^{(n)}-f\right)+j \varphi_{b}^{(n)}\right]
\end{aligned}
$$

where $t_{d}^{(n)}=t^{(n)}-t_{s}$ and

$$
f_{a}^{(n)}=t_{d}^{(n)} S_{0}=\left(t^{(n)}-t_{s}\right) S_{0}=\frac{2\left(d^{(n)}-D\right)}{c} S_{0}
$$

and

$$
\varphi_{b}^{(n)}=\pi t_{d}^{(n)}\left(2 f_{1}-S_{0} t_{d}^{(n)}\right)
$$

The frequency value, $f_{a}^{(n)}$, defined in (5), is called the position frequency ${ }^{[4]}$ of the fsm spectrum. This frequency is proportional to the difference between the actual range of the particle, $d^{(n)}$, and the measurement range, $D$.

When $\tilde{G}_{a}^{(n)}(f)$ in (4) has been obtained from the received signal, the subsequent processing is done along the same lines as for the PW-tsm Doppler system: A segment around $f=0$ of width $f_{w}$ is isolated from the $f_{s m}$ spectrum, $\tilde{G}_{a}^{(n)}(f)$, and then cross-correlated with the subsequent $f s m$ spectrum, $\tilde{G}_{a}^{(n+n)}(f)$. It is seen from (4) and (5) that consecutive magnitude $f s m$ spectra are displaced by an amount

$$
\Delta f_{a}=f_{a}^{(n+1)}-f_{a}^{(n)}=\frac{2 v T_{r}}{c} S_{0}=\tau_{0} S_{0}
$$

The spectral shift, $\Delta f_{a}$, is equal to the change in round trip travel time, $\tau_{0}$, to the scatterer multiplied with the time to frequency conversion factor, $S_{0}$. Specifically, it is seen that

while

$$
\left|\tilde{G}_{a}^{(n+1)}(f)\right|=\left|\tilde{G}_{a}^{(n)}\left(f-\Delta f_{a}\right)\right|
$$

$$
\tilde{G}_{a}^{(n+1)}(f) \neq \tilde{G}_{a}^{(n)}\left(f-\Delta f_{a}\right)
$$

The latter inequality is due to the fact that the time signals from which $\tilde{G}_{a}^{(n)}(f)$ and $\tilde{G}_{a}^{(n+1)}(f)$ are generated are not only shifted in frequency but also in time causing the phase of 
$\tilde{G}_{a}^{(n)}(f)$ to be shifted relatively to the phase of $\tilde{G}_{a}^{(n+1)}(f)$. This can be observed by comparing plot (d) with plot (e) in Figure 2. Also note that the rate of oscillation of the $f s m$ spectrum is changed by a small amount. The phase shift between consecutive $f s m$ spectra means that the unique waveform signature present in received PW signals at a given range is not preserved in the real nor imaginary part of $\tilde{G}_{a}^{(n)}(f)$. However, it is nevertheless possible to cross-correlate the real parts of $\tilde{G}_{a}^{(n)}(f)$ and from the analytic expression of this cross-correlation function derive an expression for the peak location. Note that this expression depends on the envelope of the received sweep signals. In this paper, a Gaussian envelope is assumed, as shown in (1). In an experimental situation with finite bandwidth transducers, the envelope of the excitation waveform may be chosen so that the received sweep signal from a point scatterer has the desired envelope. A good approximation to the global maximum of this cross-correlation function, for the case when the scatterer velocity is below the aliasing velocity limit $(=$ $\left.c /\left(4 f_{0} T_{r}\right)\right)$ has been found to be $\mathrm{e}^{[5]}$

$$
\gamma_{0} \cong \frac{2 T_{r}}{c}\left(S_{0}+\frac{2 f_{1}}{t_{m}}\right) v=\frac{4 T_{r}}{c} \frac{f_{0}}{t_{m}} v
$$

An estimate of $\gamma_{0}$, based on actual data, is found as

$$
\hat{\gamma}=\underset{\gamma}{\operatorname{argmax}}\left\{\hat{C}_{F M}^{(n, n+1)}(\gamma)\right\}
$$

where $\hat{C}_{F M}^{(n, n+1)}(\gamma)$ is the cross-correlation function between $\tilde{G}_{a}^{(n)}(f)$ and $\tilde{G}_{a}^{(n+1)}(f)$. Using (10) and (11), an estimate of the particle velocity can be found. An example showing the peaks of the magnitude and real parts of $\hat{C}_{F M}^{(n, n+1)}(\gamma)$ is given in Figure 3. Figure 3 illustrates the advantage of cross-correlation of the real part of the $f s m$ spectra over magnitude of fsm spectra: The cross-correlation peak is much sharper, leading to a more precise determination of velocity. The "cost" of this approach is a more complex,

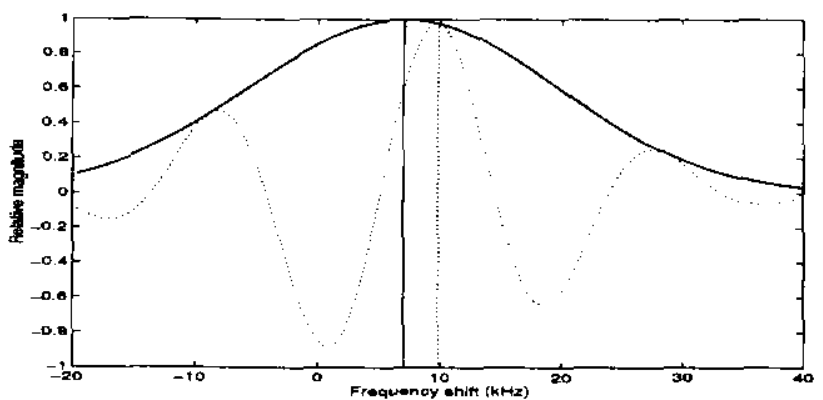

Figure 3 Magnitude ( - ) and real part (") of typical cross-correlation function in the FM-fsm Doppler system with their respective peaks indicated. envelope-dependent expression for the global maximum of the cross-correlation function.

When the FM Doppler system is implemented to provide a velocity profile, the velocity for a set of measurement ranges, $D_{\mathrm{i}}$, must be found. In the implementation presented in this paper, each range must be treated individually, requiring $\mathrm{a}$ new $D$ and FT per velocity estimate or range cell. More efficient approaches are possible, but these are beyond the scope of this paper.

\section{SIMULATION STUDIES}

This section provides a limited performance comparisons between the FM- $f s m$ and the PW-tsm Doppler systems. Specifically, a 2D flow situation with parabolic flow in a rigid tube, was modeled. The received signals were calculated from a large number of scattering centers randomly distributed inside the region from which backscattered signal would be received. The lateral variation in ultrasound beam intensity is modelled as a Gaussian shape.

The parameters identical in both system were as follows: $f_{0}=\left(f_{1}+f_{2}\right) / 2=5 \mathrm{MHz}$, the $r m s$ bandwidth is 1 $\mathrm{MHz}$ and $\alpha=3 ; T_{r}=100 \mu \mathrm{s} ; v_{\text {atias }}=0.75 \mathrm{~m} / \mathrm{s} . N=2$ and SNR $=20 \mathrm{~dB}$. The flow velocity in the center of the tube in direction of the beam was: $v_{\max }=0.85 v_{\text {atias }}=0.64 \mathrm{~m} / \mathrm{s}$. The parameters for the PW Doppler system were: $t_{m, P W}=$ $637 \mathrm{~ns}=3.2 / f_{0}$. The specific parameters for the FM Doppler system were: $f_{1}=0.5 \mathrm{MHz}$ and $f_{2}=9.5 \mathrm{MHz}$; $t_{m, F M}=0.8 T_{r}=80 \mu \mathrm{s}$.

The results of 3000 independent repetitions of the simulations is given in Figure 4 which shows the mean velocity profiles together with \pm one standard deviation for the FM and PW Doppler systems, respectively.

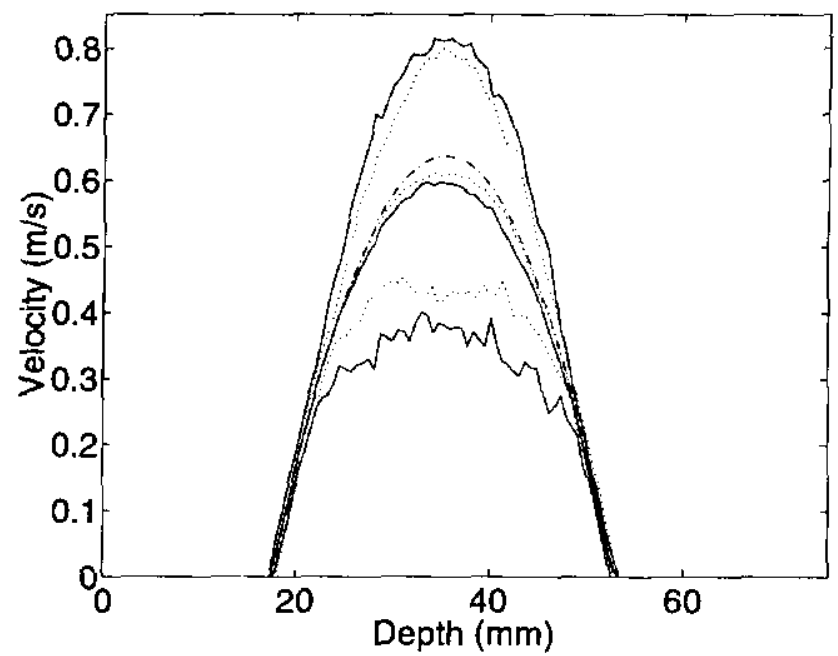

Figure 4 Mean velocity profiles shown with \pm one standard deviation for the FM- $f$ sm Doppler system (-) and the PW-tsm Doppler system (-...). The true velocity profile, TVP, (known a priori) is shown as (-'-) 


\section{EXPERIMENTAL RESULTS}

An experimental Doppler system has been developed, for the purpose of making both FM-fsm and PW-tsm Doppler measurements. The measurement system consists of: (1) a DOS based computer; (2) an arbitrary function generator for generation of the emitted PW or FM signal in analog form; (3) a power amplifier, driving the emitting transducer; (4) a focused dual element annular array ultrasonic transducer, for simultaneous emission and reception of ultrasound and (5) a digital storage oscilloscope (DSO), with which the received signal was digitized (at 8 bits resolution) and transferred to the computer. The flow phantom used a centrifugal pump that circulated a mixture of water and corn starch (2-5\% vol.) though a $20 \mathrm{~mm}$ in diameter tube.

The bandwidth of the FM system was slightly higher than that of the PW system, and the signal to noise ratio (SNR) of the analog signals was better than $40 \mathrm{~dB}$ for both systems. However, as the annular array transducer was not optimized for simultaneous emission and reception, the experimental system suffered from serious cross-talk when FM signals were emitted. Most of the cross-talk, as well as the stationary echoes from the tube wall, was removed by means of a stationary echo canceller (SEC). The SEC operated on the digitized signals in the computer, by subtracting consecutive received rf signals. For the FM-fsm Doppler system the stationary echo cancelled signals were only represented by a few bits, reducing the SNR to below $20 \mathrm{~dB}$. In the choice of system parameters, it was desired to influence the flow components of the received signals as little as possible by the SEC transfer function. At the same time $T$, was set as low as possible to maximize the number of received signals that could fit into the DSO memory. This required that rather high flow velocities were used.

All the measured velocity profiles exhibited a reasonably good agreement with the directly measured volume flows. Typical profiles obtained with the FM-fsm

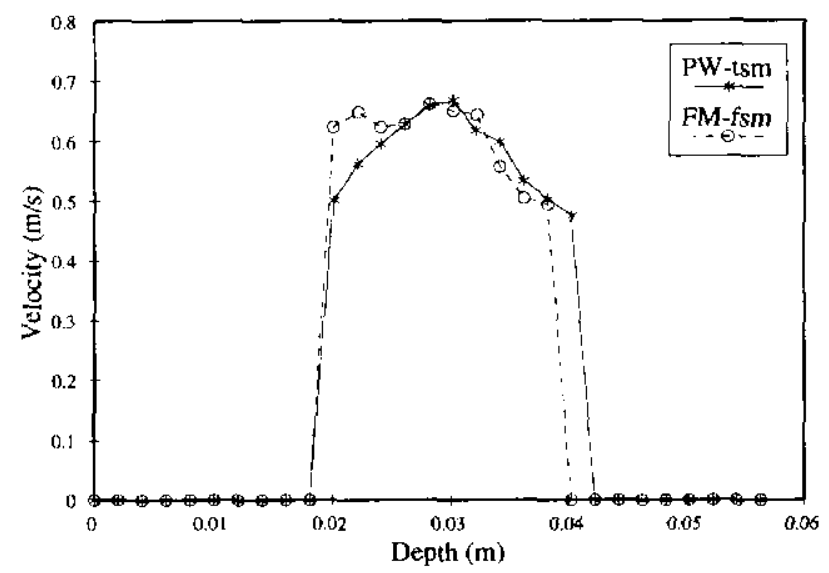

Figure 5 Measured velocity profiles for PW-tsm and FM-fsm Doppler systems.
Doppler system and PW-tsm Doppler system are shown in Figure 5. As the Reynolds number far exceeds the limit for laminar flow, the profiles tend towards a general rectangular shape, but with fluctuations, as has also been demonstrated with laser Doppler velocimetry and with bubble visualization ${ }^{[2]}$.

\section{CONCLUSIONS}

A brief description of the signal processing in the improved FM Doppler system has been given, and the technique for cross-correlating real spectral has been presented. The performance of the FM Doppler has been evaluated numerically and experimentally. In the latter, the FM and PW Doppler systems were compared using a simple flow model under semi-realistic conditions. Despite considerable cross-talk in the FM Doppler system, the flow profiles obtained with the two systems agreed quite well. In order to validate the applicability in the area of medical diagnostic ultrasound, experiments with a soft tissue-like coupling medium and in-vivo experiments need to be carried out using a transducer with lower cross-talk.

\section{ACKNOWLEDGEMENTS}

The authors would like to thank Uno Junghans and Jørn Lund, Brüel \& $\mathrm{Kjær} A / S$, for help with respect to computer solutions. This work was partly supported by the National Science Foundation under grant BCS9009886 and by the following Danish foundations: Forskerakademiet, Thomas B. Thriges Fond, V. S. Tranes Fond and H. C. Ørsteds Fond.

\section{REFERENCES}

[1] D. Dotti et al:: Blood flow measurements by ultrasound correlation techniques. Energia Nucleare, vol. 23, no. 11, pp. 571 - 575, November 1976.

[2] D. Y. Goswami: Velocity profiles of Liquid Flow through Circular Tubes and how they affect Flow measurement. Journal of Solar Energy Engineering, Vol. 113, pp. $206-210,1991$.

[3] J. E. Wilhjelm and P. C. Pedersen: Target Velocity Estimation with FM and PW Echo-Ranging Doppler Systems, Part I - Signal analysis. IEEE Trans. Ultrason., Ferroelec., Freq. Contr., vol. 40, no. 4, pp. 366 - 372, July 1993.

[4] J. E. Wilhjelm and P. C. Pedersen: Target Velocity Estimation with FM and PW Echo-Ranging Doppler Systems, Part II - Systems analysis. IEEE Trans. Ultrason., Ferroelec., Freq. Contr., vol. 40, no. 4, pp. 373 - 380, July 1993.

[5] J. E. Wilhjelm and P. C. Pedersen: Analytical and experimental comparisons between the FM- $f s m$ and the PW-tsm Doppler systems. Submitted to Journal of the Acoustic Society of America, 1995. 